\section{Forum of the European Societies of Clinical Chemistry and Laboratory Medicine}

\author{
A. M. Gressner ${ }^{1}$ und O. A. Gressner ${ }^{2}$ \\ ${ }^{1}$ Labor Dr. Wisplinghoff Berlin, Berlin, Deutschland \\ ${ }^{2}$ Labor Dr. Wisplinghoff Köln, Köln, Deutschland
}

\section{Synonym(e) FESCC}

Definition FESCC versteht sich als regionale Subgruppe der International Federation of Clinical Chemistry (IFCC), deren Mitglieder die europäischen wissenschaftlichen Fachgesellschaften für Klinische Chemie und Laboratoriumsmedizin sind.

Beschreibung Unter der Schirmherrschaft von IFCC $(\triangleright$ International Federation of Clinical Chemistry and Laboratory Medicine) agiert FESCC als europäische Task Force von IFCC, indem sie die Belange von Klinischer Chemie und Laboratoriumsmedizin sowohl global als auch auf einer regionalen europäischen Ebene vertritt. Im Einzelnen sind die Ziele:
- Fort- und Weiterbildung von Spezialisten in Klinischer Chemie und Laboratoriumsmedizin sowie Austausch von Studenten und Wissenschaftlern

- Förderung der Kooperation Klinisch-Chemischer und Laboratoriumsmedizinischer Fachgesellschaften zwischen den europäischen Ländern

- Unterstützung von Laboratoriumsakkreditierungen und -zertifizierungen und von Qualitätskontrollmaßnahmen auf europäischer Ebene

- Förderung der IFCC-Aktivitäten in Europa

- Vertretung von Klinischer Chemie und Laboratoriumsmedizin in der Diskussion mit anderen spezifischen medizinischen Laboratoriumsdisziplinen

Dem Executive Board gehören 5 Personen (ein Präsident), dem Council jeweils 2 delegierte Vertreter aller europäischen Mitgliedsgesellschaften an.

Das offizielle Publikationsorgan von FESCC ist „Clinical Chemistry and Laboratory Medicine“.

FESCC und EC4 (European Communities Confederation of Clinical Chemistry) gingen 2007 in EFCC (European Federation of Clinical Chemistry and Laboratory Medicine) auf. 